\title{
Modelo operativo de sistemas comunales de prevención de drogas: la experiencia de Chile ${ }^{1}$
}

\author{
Caris, L.*; Selamé, T.**; Agurto, M ${ }^{a}$ I.***2 \\ * Médico Psiquiatra. PhD. in Public Health, Académico Universidad de Chile. \\ * Socióloga, Doctora en Ciencias Sociales del Trabajo. \\ ** Socióloga, Doctra en Ciencias Sociales.
}

Enviar correspondencia a:

Prof. Luis Caris. Escuela de Salud Pública. Facultad de Medicina. Av. Independencia. 939 Santiago (Chile). Icaris@minsal.cl

\section{RESUMEN}

Este artículo presenta la implementación de Sistemas Comunales de Prevención utilizando el Modelo Operativo, que fue desarrollado por académicos de la Universidad de Chile para desarrollar trabajo preventivo en distintas comunas de Chile. Se presenta las etapas del Modelo Operativo el cual se basa en el trabajo en red social. Estas fueron cuatro 1. Dinamización Institucional, 2. Dinamización Reticular, 3. Dinamización Comunitaria y 4. Evaluación. Se puso en práctica en 15 comunas del país desde 1997 a 1999. Se pudo establecer que en 12 de ellas conformaron un Comité Coordinador. El municipio fue el Centro Estratégico y en 11 de ellas tuvieron diversos grados de apoyo. Una de las acciones mas importante fue la elaboración y desarrollo de proyectos de prevención a nivel local, lo cual se presentó en 13 de las 15 comunas. Se manifiestan algunos problemas como la poca integración a los comités coordinadores de personas de las comunidades y la necesidad de un mayor apoyo de las autoridades municipales y que en pocas comunas se pudo introducir el plan comunal de prevención en el Plan de Desarrollo Comunal.

Palabras claves: prevención comunitaria, modelo operativo, prevención de drogas, redes sociales, Chile.

\section{INTRODUCCIÓN}

n 1997, el Programa de Prevención de Drogas a Nivel Comunitario de la Universidad de Chile, el Consejo Nacional para el Control de Estupefacientes (CONACE) y la División de Organizaciones

\section{SUMMARY}

This article presents the implementation of a prevention program at Communal level, using the Operative Model that was developed for academics of the University of Chile to develop preventive work in different communities of Chile. The stages of the Operative Model are based in working the social network. There are four stages: 1. Institutional phase, 2. Reticular phase, 3. Community phase, and 4. Evaluation. They put into in practice in 15 communes of the country from 1997 to 1999. In 12 out of 15 communities they conformed a Coordinating Committee. The municipality was the Strategic Center and in 11 of them they had diverse degrees of support. One of the important actions it was the elaboration and development of prevention projects at local level, that were presented in 13 of the 15 communes. Some problems arose like the little integration among community leaders and people at Municipal level and the need of an stronger support from the municipal authorities. Only in a few communes we could introduce the communal prevention plan into the Communal Development Plan.

Key words: community prevention, operational model, communal systems, drug prevention, social networks, Chile.

Sociales (D.O.S.) del Ministerio Secretaría General de Gobierno, dieron comienzo a un proceso de instalación de sistemas comunales de prevención basándose en un modelo de intervención socio-comunitaria conocido como Modelo Operativo ${ }^{3}$. El proceso debía

' La preparación de este documento ha sido posible gracias al aporte del Departamento de Investigación y Desarrollo de la Universidad de Chile, Proyecto No EDID99/019.

${ }^{2}$ Los autores agradecen los aportes efectuados a esta publicación por: María Aragonés J., Ana Marchandón A., Christian Pereda F., Fanny Serani y Marianela Varas $P$.

${ }^{3}$ Ver Consejo Nacional para el Control de Estupefacientes, Vicerrectoría de Asuntos Académicos y Estudiantiles de la Universidad de Chile, y División de Organizaciones Sociales del Ministerio Secretaría General de Gobierno: Sistema Comunal de Prevención de Drogas. Modelo Operativo, Santiago de Chile, sin fecha. 
finalizar en septiembre de 1999. El modelo (en adelante M.O.), había sido aplicado en 1995/1996 en forma experimental en tres comunas de la Región Metropolitana de Santiago. La experiencia fue sometida a evaluación y de ella surgió una versión definitiva del M.O., versión que es la que actualmente se conoce y que se continúa aplicando en varias comunas del país.

El equipo de profesionales del Programa de Prevención de Drogas de la Universidad de Chile ${ }^{4}$, junto con los organismos públicos antes señalados, inició en 1997 la aplicación piloto en 15 comunas (ubicadas en 8 diferentes Regiones) del país, de igual número de sistemas de prevención estructurados en red que seguían las directrices entregadas por el M.O. En lo específico, mientras la labor del CONACE (a través del nivel central y de sus instancias regionales-CORECEs) y de la División de Organizaciones Sociales consistía en financiar, supervisar y promover en los sectores institucionales y ante las autoridades comunales la instalación del Modelo, la Universidad de Chile cumplía, en tanto, labores de asesoría, de apoyo técnico y de capacitación.

El presente trabajo entrega una visión sucinta de los principales hitos que marcaron la aplicación práctica del Modelo en las comunas seleccionadas, y que determinaron en definitiva sus niveles de logro. Especial énfasis se ha colocado en el análisis del "desempeño" real que tuvieron actores considerados claves, entre los que se incluyen tanto personas individuales (fundamentalmente el Alcalde y el/la encargado/a comunal de la implementación del sistema), como el de instancias representativas del sector institucional y el de las organizaciones sociales y comunitarias.

\section{EL MODELO OPERATIVO}

El Modelo Operativo es una estrategia para la acción que privilegia "la formación y desarrollo de redes comunales de prevención mediante la instalación de capacidades de gestión, coordinación, formulación y evaluación de proyectos en organizaciones y actores de los ámbitos institucional y comunitario" 5 . Toma como punto de partida, por un lado, la adecuada articulación en red de las diferentes instancias sectoriales institucionales (dependencias gubernamentales, Municipio, iglesias, organismos no gubernamentales con experiencia en el trabajo preventivo). Luego de lograda la organización intersectorial, debe producirse el trabajo en red entre éstas y las organizaciones comunitarias.
Para la instalación del sistema comunal de prevención (SISCOP), el M.O. propone: una forma de organización del conjunto de agentes institucionales y comunitarios que actuarán en la red (al nivel comunal), y una secuencia de acciones tendientes a ella.

\subsection{Forma de Organización para la Acción}

Como forma de organización para la acción, el M.O. propone la conformación de una Red Social Comunal. Ésta, a su vez, tiene: i) un Centro Estratégico, que es el Municipio; y ii) un Centro Operativo o Comité Coordinador.

En torno al Comité Coordinador se articulan los Conglomerados, que son unidades territoriales más pequeñas que la comuna (barrio, manzana, unidad vecinal) que tienen una identidad social como grupo de población especial, presentando características geográficas, culturales o laborales específicas.

El Modelo Operativo propende a que en los conglomerados se vinculen organizaciones y actores provenientes de los ámbitos institucional (sectores salud, educación, iglesias, Oficinas de la Juventud, organizaciones no gubernamentales, etc.) y comunitario (Uniones Comunales de Juntas de Vecinos, Centros Culturales, Clubes Deportivos, etc.).

Las 3 instancias: Municipio (Centro Estratégico), Comité Coordinador (Centro Operativo), y conjunto de conglomerados, conforman el sistema comunal de prevención.

- El Centro Estratégico está conectado a los organismos del nivel Central (Ministerios, CONACE), del nivel Regional (Secretarías Regionales Ministeriales- SEREMIS, Intendencias, CORECEs, etc.), y del nivel Provincial (Gobernaciones, COPROCEs, etc.).

- Por su parte, el Comité Coordinador consta de 4 componentes cada uno de los cuales asume funciones diferenciadas pero no excluyentes que tienen que ver con:

-Gestión. Este Componente es el encargado de conseguir recursos para el financiamiento de la Red, coordinar las actividades de la Red, dirigir la evaluación de las actividades, seleccionar los proyectos comunitarios que serán financiados a través de fondos concursables.

-Comunicación. Componente cuya función es servir de enlace entre todos los integrantes de la Red, y entre la Red y la comunidad, a fin de divulgar las diferentes actividades que ella realiza.

\footnotetext{
${ }^{4}$ El Programa de Prevención de Drogas está adscrito a la Escuela de Salud Pública de la Facultad de Medicina de la Universidad de Chile.

${ }^{5}$ Consejo Nacional para el Control de Estupefacientes, Vicerrectoría de Asuntos Académicos y Estudiantiles de la Universidad de Chile, y División de Organizaciones Sociales del Ministerio Secretaría General de Gobierno: Sistema Comunal de Prevención....; p.5.
} 
-Capacitación. Toma a su cargo el conjunto de acciones vinculadas a la puesta en marcha de un curso de capacitación que entregará herramientas y conocimientos sobre prevención a los actores institucionales y comunitarios que conforman la Red. Evaluación y Planificación. Su objetivo es efectuar el seguimiento permanente de las acciones que está llevando a cabo la Red, y apoyar al resto de los Componentes con la información sistematizada en cada etapa.

\subsection{Etapas y Sub-etapas}

El M.O. propone una secuencia de acciones que comprende, por un lado, Grandes Etapas o Módulos, $y$, por otro lado, sub-etapas.

Un Módulo es un conjunto de actividades organizadas en torno a un propósito global, cuyo desarrollo estará condicionado a la realidad que vive cada comuna. Cada Módulo puede ser organizado en sub-etapas, y estas sub-etapas estarán orientadas a activar organizaciones comunales. Las 4 grandes Etapas que distingue el Modelo Operativo son:

ETAPA I DE DINAMIZACIÓN INSTITUCIONAL: su objetivo es lograr la articulación en red de un conjunto de instituciones en torno a la prevención del consumo indebido de drogas en el espacio comunal. Gestión de red es la estrategia predominante. Es una etapa de establecimiento de acuerdos y compromisos institucionales para la implementación del Sistema Comunal.

En esta etapa son involucrados para el trabajo en Red los agentes institucionales del nivel comunal, provincial y regional cuidando que estén bien representados los siguientes ámbitos: Consejo Nacional para el Control de Estupefacientes, Salud, Educación, Justicia, Carabineros, Investigaciones, Autoridades Religiosas, Organizaciones No Gubernamentales.

Básicamente, ésta es una etapa abocada al trabajo de persuasión, de sensibilización, de motivación en torno a los postulados centrales del trabajo en Red.

Como el Trabajo en red debe articularse por conglomerados, en esta etapa se identifican sectores geográficos (poblaciones, barrios, sectores), de acuerdo a la visión que la comunidad tiene de sus espacios de vida. Se procede a hacer un listado por conglomerados de los representantes del Sector institucional, y de sus principales responsables (colegios, jardines infantiles, consultorios, iglesias, comisarías, organismos no gubernamentales). Asimismo, se hace un listado de actores que tienen responsabilidades formales en el ámbito comunitario: Uniones Comunales de Juntas de Vecinos, Juntas de Vecinos, grupos culturales, centros de madres, clubes deportivos, sindicatos, etc.

Con estos datos (conglomerados definidos, listado de organismos institucionales, listado de organizaciones comunitarias), se convoca a los actores a conformar una Red de Prevención que es el propósito del MÓDULO O ETAPA II.

ETAPA II DE DINAMIZACIÓN RETICULAR: su objetivo es articular en red a las organizaciones institucionales y comunitarias e iniciar el proceso de conformación de la red. El énfasis está puesto en la conformación de redes sociales más pequeñas que la comuna, en base a la información recopilada en el Módulo I. El trabajo de cada uno de los Componentes, en especial el de Capacitación (el cual, a su vez, involucra la elaboración, evaluación, aprobación de proyectos de prevención del nivel comunitario), debe estar orientado por este objetivo. Se debe producir el encuentro entre actores comunitarios e institucionales. Las actividades centrales asociadas a esta Etapa son la conformación de redes micro-locales de prevención, la convocatoria de actores, la producción de encuentros en red flexibles y creativos entre actores institucionales y actores comunitarios, la realización de diagnósticos micro-locales, la capacitación de los actores y la elaboración de proyectos comunitarios de prevención.

ETAPA III DE DINAMIZACIÓN COMUNITARIA: sU objetivo es lograr la articulación en red de un conjunto de actores y organizaciones comunitarias en torno a un plan y en torno a la ejecución de proyectos comunitarios de prevención en sus espacios de vida. En este Módulo la actividad principal es la ejecución por parte de los actores comunitarios de los proyectos elaborados en la etapa anterior, con la asesoría y apoyo de los actores institucionales de los respectivos conglomerados. El objetivo de este Módulo se logra a través de la asignación de recursos para la ejecución de los proyectos comunitarios que fueron elaborados en el proceso de capacitación.

A su vez, uno de los objetivos centrales de los proyectos comunitarios es lograr el fortalecimiento de los conglomerados o redes locales de prevención. Es a través de estos proyectos como los conglomerados se conectan directamente con las personas y grupos afectados directamente o indirectamente por el problema del consumo indebido de alcohol y drogas.

ETAPA IV DE EVALUACIÓN: el objetivo de esta Etapa es evaluar los resultados de la implementación del Modelo Operativo y elaborar un Plan Comunal de Prevención. Para ello se debe organizar un evento que reúna a todos los actores y organizaciones locales integradas a los diferentes conglomerados a fin de que procedan a la evaluación del funcionamiento del sistema comunal de prevención y se establezcan las bases de un Plan Comunal de Prevención. En esta 
Etapa se efectúan las gestiones para que dicho Plan sea integrado al Plan de Desarrollo Comunal (PLADECO).

Los encargados de cada Componente elaboran un informe Ejecutivo sobre su gestión.

Cabe señalar que el M.O. pone a disposición de los actores una serie de instrumentos tendientes a facilitar el desarrollo de las diversas actividades. Éstos van, desde formatos para acopiar información (encuestas, fichas de inscripción de participantes, pautas para efectuar catastro de organizaciones sociales, entre otros) hasta cartillas sobre contenidos relativos a la instalación de sistemas comunales de prevención, pasando por modelos de cartas para llamar a concursos de presentación de afiches.

\section{CRITERIOS DE EVALUACIÓN}

El trabajo en red, la participación activa de las organizaciones comunitarias y del sector institucional, la articulación entre sectores institucional y comunitario, la asunción por parte del Municipio de un rol clave en la puesta en funcionamiento del sistema ${ }^{6}$, constituyen, entre otros elementos, los pilares sobre los cuales se basa la estrategia postulada por el M.O. para la implementación de sistemas comunales de prevención.

De los antecedentes entregados en relación con la estrategia propuesta por el M.O. puede colegirse la importancia que reviste para la instalación de un sistema de prevención al nivel comunal el adecuado desempeño del Municipio en términos de: a) interés por encarar el tema de los factores de riesgo y las conductas no deseables vinculadas al consumo abusivo de drogas; b) disposición para trabajar en red; c) buena disposición por parte de éste y del sector institucional para apoyar iniciativas que emerjan de las diferentes instancias que trabajan en el nivel comunal y/o que pueden aportar con soluciones al problema; d) ofrecimiento de infraestructura y asignación de recursos humanos, técnicos y materiales para el funcionamiento del Comité Coordinador; e) delegación de roles y funciones en actores extra-municipio; f) apoyo en la etapa de capacitación de los actores y de dinamización comunitaria; g) defensa por parte del Alcalde ante el Concejo Municipal de la idea de incorporar el plan de prevención comunal - o, a lo menos, el tema de la prevención -, al Plan de Desarrollo Comunal (PLADECO).
A fin de poder concluir acerca de la capacidad que el M.O. presenta para montar, desarrollar y sostener sistemas comunales de prevención, se ha procedido a evaluar lo logrado por las instancias públicas que tuvieron un rol protagónico en la experiencia de instalación de SISCOP en 15 comunas del país en el período 1997-1999. Para ello, fueron seleccionados 6 ítems, de los cuales 3 aluden al papel que en el proceso jugó el Municipio - centro estratégico del sistema -, en tanto los restantes 3 dan cuenta del grado de participación de los dos sectores - institucional y comunitario -, y de coordinación alcanzado entre ellos. Los ítems son:

\section{-SOBRE “DESEMPEÑO” DEL MUNICIPIO}

a) Funcionamiento y grado de estructuración del Comité Coordinador;

b) Apoyos más importantes del Municipio a implementación del SISCOP (horas funcionarias, infraestructura, equipamiento, etc.);

c) Incorporación tema prevención al PLADECO;

-SOBRE PARTICIPACIÓN COMUNITARIA E INSTITUCIONAL

a) Grado de articulación entre los sectores institucional y comunitario;

b) Representación de las organizaciones comunitarias en la Red de Prevención (distribución de funciones entre sectores institucional y comunitario);

c) Presentación de proyectos comunitarios.

La información pertinente sobre cada comuna fue extraída del Informe elaborado en 1999 por el Programa de Prevención de Drogas de la Universidad de Chile para el Ministerio del Interior (CONACE) y para el Ministerio Secretaría General de Gobierno (D.O.S.). En él se hace un recuento de las circunstancias particulares que marcaron el proceso de instalación en cada comuna de un sistema comunal de prevención basado en la estrategia propuesta por el Modelo Operati$\mathrm{Vo}^{7}$. Este documento consta de 15 informes comunales que fueron desarrollados por los responsables del Programa siguiendo una pauta de referencia pre-establecida.

\section{PRINCIPALES RESULTADOS}

A continuación se presenta una síntesis que contiene los resultados que se pueden deducir del análisis secundario efectuado de los Informes Comunales.

\footnotetext{
${ }^{6}$ Cabe señalar que el M.O. admite que otras organizaciones o sectores puedan sustituir al Municipio en su rol de "centro estratégico" del SISCOP, en aquellos casos en que éste no pueda asumirlo.

${ }^{7}$ Ver Informe Final de Proceso. Sistema Comunal de Prevención del Consumo de Drogas a Nivel Comunitario: Un Modelo Operativo 1997-1999, Santiago de Chile, octubre de 1999.
} 
Se da cuenta de ellos abordando por separado cada aspecto de los mencionados anteriormente.

\section{a) Funcionamiento y grado de estructuración del Comité Coordinador}

En lo que compete a la conformación, funcionamiento y grado de estructuración del Comité Coordinador, centro operativo y motor del proceso de instalación del sistema comunal de prevención, se pudo detectar que en 2 de las 15 comunas en las cuales se desarrolló la experiencia bajo análisis hubo resistencia a la implementación del SISCOP en la modalidad propuesta por el M.O. En estas comunas se detectó baja comprensión de la conceptualización y metodología del M.O. Si se realizó trabajo preventivo en ellas fue siguiendo esquemas muy ajenos a éste.

En una tercera comuna se estructuró un Comité Coordinador sui géneris, que no ajustaba estrictamente a los lineamientos establecidos por el M.O.

En la totalidad de los 12 municipios que sí conformaron Comité Coordinador se pudo constatar la centralización del proceso de toma de decisiones radicándose éste en uno o más funcionarios designados por la máxima autoridad municipal, el Alcalde. La incorporación de los sectores institucionales en el Comité alcanzó diversos niveles en las diferentes comunas, aunque usualmente se registró la incorporación de un número limitado de éstos. Problemas asociados a la falta de autorización y reconocimiento de la labor desarrollada en esta instancia por parte de los jefes directos de los representantes del sector institucional, así como la tendencia a a la concentración de responsabilidades y funciones en la persona del/de la encargado/a municipal (que ejercía la función de coordinación y sistematización del Comité Coordinador), se constituyeron en factores que provocaron desmotivación en estos agentes.

Rara vez fue incluido un representante del sector comunitario en el Comité Coordinador en calidad de responsable de alguno de los 4 componentes de la acción: Gestión, Comunicación, Capacitación y Evaluación y Planificación.

En 9 de las 12 comunas en que se estructuró un Comité Coordinador se pudo percibir debilidad orgánica y de funcionamiento, que se traducía en participación irregular de sus integrantes, rotación de éstos, asistematicidad en la convocatoria a reuniones, escasa actividad concreta o bien concentración de las responsabilidades en unos pocos miembros. Sin duda, diversos factores como la falta de capacidad de liderazgo y/o de suficiente motivación para trabajar en un sistema de prevención en red demostrado por algunos/as de los/as encargados/das designados por el Alcalde, la sobrecarga laboral de éstos, las prácticas poco democráticas de interrelación con el resto de los integrantes del Comité Coordinador, así como, también, la falta de reconocimiento formal de la labor realizada por los agentes institucionales en este Comité, conspiraron contra una mayor estructuración y un mejor funcionamiento de esta instancia.

\section{b) Apoyos más importantes del Municipio a implementación del SISCOP}

El grado de apoyo prestado por el Municipio al proceso de instalación del SISCOP fue disímil entre una comuna y otra. Puede decirse que éste alcanzó su expresión máxima en una comuna que puso a disposición del Comité Coordinador una no despreciable suma de dinero, horas funcionarias de los niveles profesional y administrativo, defensa del proyecto de instalación del SISCOP ante el Concejo Municipal, y participación en calidad de expositor en las actividades de capacitación implicadas en el proceso. En el otro extremo, se verificó el caso de 4 comunas en las que no se otorgó apoyo.

La vía más recurrente de colaboración de las restantes comunas fue la asignación de horas laborales del/de la encargado/a para que las destinase a la gestión del Comité Coordinador y la contratación de horas profesionales para colaborar en la elaboración y/o ejecución de los proyectos comunitarios.

Cabe destacar que este aporte, por lo general, no fue gratuito, porque contribuyó a la "municipalización" del proceso, es decir, otorgó más "argumentos" al Municipio para reforzar su tendencia a la centralización del proceso de toma de decisiones en la persona del encargado comunal o en autoridades superiores a él.

\section{c) Incorporación tema prevención al PLADECO}

Como fuera señalado en el punto II, entre las subetapas de la Fase Final (Evaluación) del M.O. está contemplada la elaboración de un plan comunal de prevención con miras a dejarlo incorporado al Plan de Desarrollo Comunal (PLADECO) del Municipio. Ello constituye un mecanismo clave para dar ciertas garantías de continuidad y estabilidad en el trabajo preventivo. La falta de logro de este objetivo ciertamente significa que los riesgos de discontinuidad del programa se incrementan, pero también está señalando la presencia de factores obstaculizadores que se hace preciso investigar.

El balance arrojado por la indagación de evidencias a este respecto es que sólo en una comuna de las 15 "intervenidas" se logró este objetivo, lo que en concreto significó que el sistema de prevención contaría con algunos recursos (humanos y financieros) durante 4 años. 
En una segunda comuna el tema preventivo se encontraba ya desde antes incorporado al PLADECO, aunque, al parecer, esta incorporación revestía un carácter más bien simbólico.

Cabe preguntarse si los resultados en este ámbito habrían sido diferentes si el proceso de instalación del SISCOP hubiese contado con un tiempo más prolongado de asesoría técnica por parte del equipo de profesionales de la Universidad de Chile. Como se ha señalado, la incorporación del tema preventivo al PLADECO constituye una de las últimas sub-etapas en la instalación del SISCOP, y, en general, las Etapas anteriores del proceso habían experimentado importantes retrasos. En un escenario en que el equipo de la Universidad de Chile tenía plazos que cumplir, y en que todo el proceso tomó mayor tiempo del planificado originalmente, bien podría esperarse que con plazos más extendidos este objetivo hubiese podido ser alcanzado.

\section{d) Grado de articulación entre los sectores ins- titucional y comunitario}

La articulación en red entre entidades institucionales y organizaciones comunitarias formales en torno a las tareas de prevención del consumo de drogas constituye, en el marco del M.O., un pre-requisito indispensable y a la vez el punto de partida de un sistema comunal de prevención en los términos postulados por el modelo.

De acuerdo con los antecedentes disponibles, más que el logro del objetivo de dejar articulados en red a ambos sectores, se registró una "toma de contacto" entre unos y otros que tuvo lugar al momento de la realización de los cursos de capacitación y también con ocasión de la ejecución de los proyectos comunitarios de prevención.

En una comuna se informó que fueron constituidos "comités coordinadores locales al nivel de conglomerados", lo que representaría una buena señal de la existencia de trabajo intersectorial. En una segunda comuna se constató la pre-existencia de tal articulación vehiculizada no por el Comité Coordinador, sino por una orgánica impulsada por el Sector Salud y con sede en los Consultorios de Salud.

En 2 comunas existiría "un contacto permanente" e intercambio de información, de conocimientos y de recursos, en general.

En otras 5 comunas hubo "incorporación" de los sectores comunitario y/o institucional a las actividades de capacitación contempladas en el M.O., y/o “integración" entre ambos sectores en el momento de la implementación de los proyectos comunitarios. Sin embargo, no resulta posible afirmar que tales conexiones y participaciones hayan perdurado y hayan quedado plasmadas en redes intersectoriales.
Por último, cabe señalar, que 6 comunas estuvieron simplemente lejos de alcanzar este importante objetivo.

\section{e) Representación de las organizaciones comu- nitarias en la Red de Prevención (distribución de funciones entre sectores institucional y comunitario)}

La participación en un trabajo preventivo conjunto de los agentes que representan a los sectores institucional y comunitario, los cuales, de acuerdo con el M.O. juegan un papel protagónico, permitiría afirmar que existe una red de prevención en una determinada comuna. Partiendo de esta premisa, se ha procedido a evaluar si los intentos emprendidos por instalar un SISCOP en las 15 comunas analizadas lograron integrar al sector comunitario.

Sólo en 5 de las 15 comunas analizadas se constató participación de las organizaciones comunitarias en el proceso de instalación del SISCOP. En algunos de estos casos, sin embargo, este logro no puede adjudicarse exclusivamente a este proceso sino a la preexistencia de redes impulsadas por el Municipio o por algún sector institucional.

En 7 comunas tal participación comunitaria no se dio. En las 3 restantes, se habría dado en forma más bien esporádica.

\section{f) Presentación de proyectos comunitarios}

En el contexto del M.O., la elaboración y ejecución de proyectos comunitarios de prevención por parte de los actores comunitarios reviste gran importancia: aparte de permitir la realización de actividades preventivas en el nivel micro-local, debe fortalecer los vínculos establecidos entre los 2 sectores en etapas anteriores, así como, también, conectar la red de prevención con la comunidad.

En lo que dice relación con el número de proyectos, pudo constatarse que 2 comunas no lograron desarrollar los mecanismos necesarios que hubiesen permitido la elaboración de proyectos comunitarios de prevención.

En el otro extremo se sitúan 2 comunas que presentaron 12 proyectos la primera, y 13 proyectos la segunda. El mínimo de proyectos presentados fue de 2 (es el caso de una sola comuna). Los proyectos aprobados en las 13 comunas que presentaron propuestas suman un total de 56, lo que arroja un promedio de 4,3 proyectos por comuna. Constituye éste un buen resultado, al cual contribuyó la asesoría prestada por los agentes institucionales presentes en la comuna y por el equipo de la Universidad de Chile. 
En este sentido, puede afirmarse que el proceso de instalación del SISCOP en 13 comunas se vio reforzado por la participación de los agentes comunitarios e institucionales en la elaboración y desarrollo de proyectos de prevención en el nivel local.

En cuanto a la naturaleza de los proyectos aprobados, primordialmente éstos estuvieron enfocados a la promoción de la sana ocupación del tiempo libre y al desarrollo de habilidades sociales por intermedio de la realización de actividades de creación artística y deportivas. En otras palabras, predominaron los proyectos asociados a una prevención inespecífica, si bien también se presentaron proyectos que a través de charlas de información y de orientación pretendían advertir a los jóvenes acerca de los riesgos que conlleva el uso abusivo de drogas.

\section{CONCLUSIONESY COMENTARIOS}

Del análisis efectuado se desprende, en primer lugar, que el Proyecto impulsado por el CONACE, la DOS y la Universidad de Chile, que estuvo encaminado a dejar instalados en 15 comunas del país sistemas comunales de prevención siguiendo las directrices del M.O., alcanzaron sólo un moderado nivel de logro. Se dejaron instaladas capacidades preventivas en las distintas comunas en que transcurrió la experiencia, así como, también, una mayor estructuración de las actividades preventivas desarrolladas y se allanó el camino hacia una relación más fluida entre municipio y sociedad civil. Sin embargo, varios de los objetivos propuestos por el M.O. para las distintas etapas de instalación del SISCOP no alcanzaron éxito en un número importante de comunas.

Los Municipios, consignados por el M.O. como los centros estratégicos en el proceso de instalación de los SISCOP, expresaron de palabra su interés en el proyecto, $y$, en importante número de casos, manifestaron este interés no sólo verbalmente, sino también en los hechos. Sin embargo, la propuesta no logró situarse con rango prioritario en el ranking de problemas que cotidianamente deben encarar los Municipios.

Por otro lado, la intervención de la administración municipal tendió a imprimir al proceso un sesgo que contraviene una de las premisas básicas que sustentan el modelo, cual es la de trabajar sobre la base de relaciones horizontales y con espíritu democrático. Esta postura, que en definitiva entorpeció tanto las relaciones entre actores institucionales y comunitarios como las sostenidas por los/las encargados/as comunales de la instalación del sistema preventivo con sus jefes directos, es, por lo demás, coherente con lo que han sido tradicionalmente las relaciones Estado-sociedad civil en nuestro país, en que una parte - el Muni- cipio - asume el rol de gestor, organizador, administrador y benefactor mientras la otra - la comunidad, juega un papel pasivo en el devenir de su comuna.

La experiencia analizada lleva a pensar en la importancia de efectuar buenos diagnósticos en torno a la realidad comunal previamente a la introducción de cualquier intento de intervención social, particularmente cuando se trata de introducir una forma de trabajo que, como el M.O., hace considerables requerimientos en cuanto a idiosincrasia, estilos de liderazgo, y normas de interrelación del Municipio y de los sectores institucionales entre sí y de ambos con la comunidad y, viceversa, de ésta con el Municipio y con los organismos del Estado.

Un rasgo que no favorece la aplicabilidad del M.O. es la dificultad semántica que encierra su terminología. De hecho, a lo menos dos comunas no lograron entender el lenguaje ni el modelo mismo, y en la mayor parte de las restantes comunas el proceso de instalación demoró más de los programado en parte, debido a problemas de baja comprensión de la terminología utilizada. Esto además de la falta de experiencia de trabajo en red por parte de los agentes municipales también aportó su cuota de dificultad.

Sin duda, en la mayoría de las comunas quedaron instaladas estructuras preventivas en red, aunque cada comuna imprimió un sello propia a las mismas, lo que tiene de importancia que a diferentes estados de desarrollo de capacidades preventivas y de apoyo de los alcaldes, utilizando este modelo operativo se pudo constituir Sistemas Comunales de Prevención a lo largo del país.

\section{BIBLIOGRAFÍA}

(1) Amtmann, Carlos A. y Liliana Larrañaga: "Los Consejos Económicos y Sociales Comunales: Posibilidad o Ficción de Participación Comunitaria Local ?", en Estudios Sociales, CPU, No 81, trimestre 3, 1994, Santiago de Chile; p. 12.

(2) Asún, D.: "Reflexiones sobre la Situación de la Prevención de las Drgodependencias en Chile", en Seminario. Análisis de las Acciones Preventivas en relación a la Política y Plan Nacional de Prevención y Control de Drogas. República de Chile, Ministerio del Interior, CONACE, Santiago, Chile; 1994.

(3) Bernstein, D. y Nietzel, M.: "Introducción a la Psicología Clínica", De. Mac Graw Hill, México; p. 481.

(4) M.Bobenrieth, C.Pereda et als "La Organización Informal: Análisis sociométrico de un Curso de Administración Hospitalaria" en Boletin de la Oficina Sanitaria Panamericana 80,5,Mayo 1976. 
(5) D.T.Campbell and JC.Stanley "Experimental and quasi-experimental Designs for Rersearch" Chicago, RAND McNally, 1963.

(6) L.Caris et als "Resultados de aplicación del cuestionario DUSI (Drug Use Screening Inventory) en establecimientos escolares de Chile y en grupos de no escolares en las comunas de Conchalí, PeñaIolen y Cerro Navia, Informe para Fondecyt.

(7) CEPAL: Transformación Productiva con Equidad, Santiago de Chile, marzo de 1990; p. 244.

(8) Costa, M. y López, E.: "Salud Comunitaria”, Biblioteca de Psicología, Psiquiatría y Salud. Serie salud 2000, De. Martínez Roca, Barcelona, 1986; p. 104

(.8)Chollat-Traquet Comunity intervention programmes en "Evaluating Tobacco Control Activities" WHO, Geneva 1996.

(9) Elina Dabas: Red de Redes, editorial Paidós, Buenos Aires, Argentina, 1993; 85.

(10) Developmental Research and Programs, Inc. CTC Prevention Strategies: A Research Guide to What Works, 1996.

(11) Fawcet et al., 1993, en Developmental Research and Programs, Inc. CTC Prevention Strategies: A Research Guide to What Works, 1996.

(12) Fuentealba, R.: “Prevención del Uso Indebido de Drogas", en Seminario. Análisis de las Acciones Preventivas en Relación a la política y Plan Nacional de Prevención y Control de Drogas, República de Chile, Ministerio del Interior, CONACE, Santiago, Chile, 1994; p. 65.

(13) Montero, M., 1984, en Unger, G.: “Orígenes y Fundamentos de la Perspectiva Comunitaria en el Quehacer de la Psicología", Revista Chilena de Psicología, Vol. 16, N², p. $3-10$.
(14) National Institute on Drug Abuse: Prevención del Uso de Drogas entre Niños y Adolescentes. Guía basada en Investigaciones, 1997.

(15) Nutbean, en Martínez, M.; León, J.; Jiménez, I. y Barriga, S: , 1992, p. 60.

(16) Organización Panamericana de la Salud/Fondo de Población de las Naciones Unidas "Evaluación de programas de salud y toma de decisiones" Washington DC, 1992.

(17) Programa de Prevención de las Fármacodependencias, Instituto Interamericano del Niño: Manual Regional de Educación Preventiva Participativa para Jóvenes y Padres. IIN O.E.A. Uruguay.

(18) "Programa, Fondo Nacional Concursable de Proyectos para la Prevención, Tratamiento y rehabilitación del Consumo de Drogas, Concurso 1998"

(19) Sánchez, A.: “Teoría y Praxis Comunitaria: Propuestas .... y Realidad", en A. Sánchez (comp.): Programas de Prevención e Intervención Comunitaria, Ediciones PPU, Barcelona, España, 1993; pp. 19 y 20.

(20) Sánchez , A: "Psicología Comunitaria. Bases Conceptuales y Operativas. Métodos de Intervención", PPU, Barcelona, 1991.

(21) Vicerrectoría de Asuntos Académicos y Estudiantiles, Universidad de Chile, Consejo Nacional para el Control de Estupefacientes y División de Organizaciones Sociales, Ministerio Secretaría General de Gobierno: Sistema Comunal de Prevención de Drogas, Modelo Operativo, Santiago de Chile 1997.

(22) TM.Wickizer,M.Von Korff,A.Cheadle et als "Activating Communities for Health Promotion: A Process Evaluation Method" en American Journal of Public Health 83,4,April 1993. 